\title{
Epigenetic targets on the table
}

Fragile X syndrome (FXS) is a neurodevelopmental disorder that lacks disease-modifying treatment options. It results from loss of function of the fragile $\mathrm{X}$ mental retardation protein (FMRP), which represses the translation of particular mRNA transcripts. A recent study in Cell has identified epigenetic regulation of transcription as an important function of proteins that are under translational control by FMRP, and has shown that pharmacological inhibition of the chromatin-associated bromodomaincontaining protein 4 (BRD4) is beneficial in FXS mouse models.

Previous studies have highlighted that FMRP regulates the translation of synaptic targets, but investigational therapies directed at these synaptic

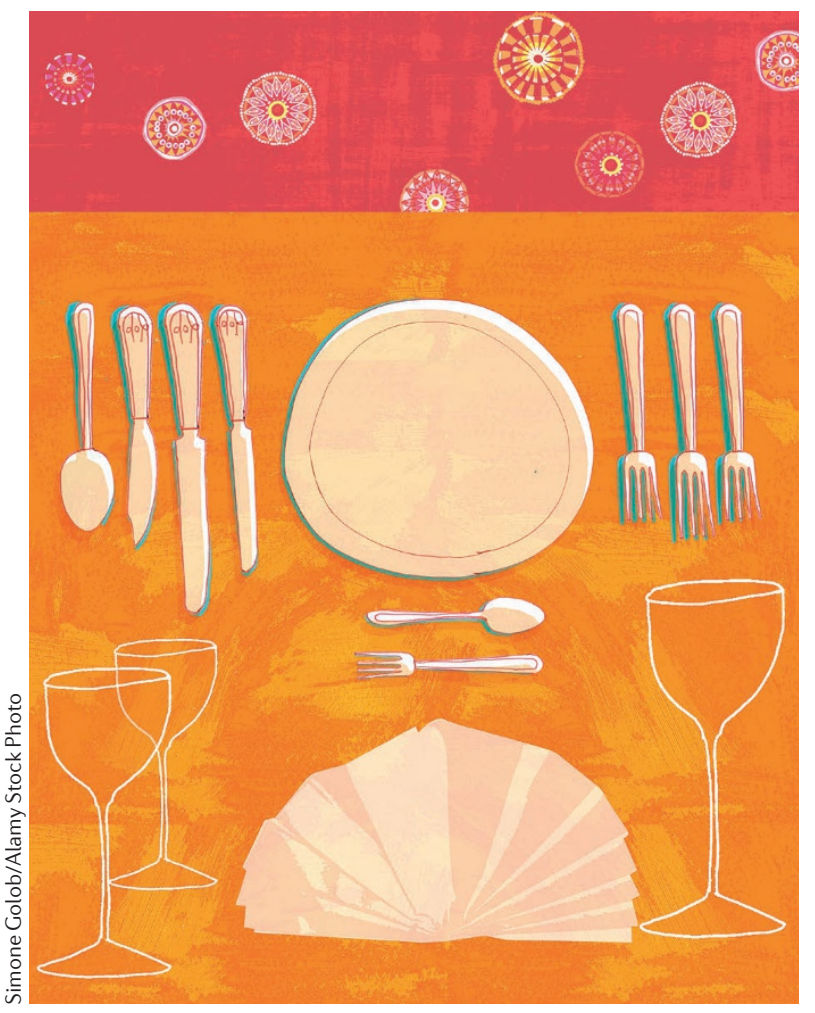

proteins have not proved fruitful. Therefore, for the current study, Korb et al. set out to identify alternative FMRP target proteins that might be involved in FXS pathophysiology.

Earlier work using nextgeneration sequencing had suggested that FMRP might also regulate the translation of proteins involved in epigenetic regulation. Such epigenetic regulators include chromatinassociated proteins, which modify the transcription of target genes by post-translational modification of histones, such as methylation. Here, Korb et al. showed that the levels of several chromatin-associated proteins, such as BRD4, were higher in cortical neurons taken from knockout mice lacking the FMRP gene $\left(\mathrm{Fmrl}^{-/-}\right.$mice) than from wildtype mice.

To assess the downstream effects of these alterations in chromatinassociated proteins, the authors used chromatin immunoprecipitation and genome-wide sequencing (ChIP-seq) and RNA sequencing. Fmr1 $\mathrm{F}^{-/-}$mice displayed widespread histone dysregulation and robust changes in gene expression, with more genes increased than decreased in neurons from $\mathrm{Fmrl}^{-/-}$mice compared with wild-type controls. Comparison of these gene profiles with published data sets on genes that are regulated by the altered chromatin-associated proteins revealed substantial overlap. The results suggest a mechanism whereby translational repression of target transcripts by FMRP modulates epigenetic regulators and, thereby, controls transcription.

Next, the authors honed in on BRD4 as a potential therapeutic target, in part because this transcriptional regulator is known to control synaptic genes and is a hub for several
FMRP targets. The investigators found that BRD4 levels were elevated in $\mathrm{Fmrl}^{-/-}$neurons relative to wildtype neurons. In vitro application of the compound JQ1, which inhibits BET proteins including BRD4, downregulated many of the overexpressed genes in $\mathrm{Fmrl}^{-/-}$neurons.

To look for potential therapeutic benefit in FXS, the team tested JQ1 in vitro on a well-established phenotype of the disease: increased spine number. They found that application of JQ1 for 24h to Fmr1 ${ }^{-/-}$neurons reduced spine number to wild-type levels. Importantly, therapeutic benefits of JQ1 were also observed in vivo: 1 week after intraperitoneal injection of JQ1, Fmr1 $1^{-/-}$mice no longer buried more marbles than did wild-type mice, indicating a reduction in perseverative and repetitive behaviour, and showed improved performance in a test of social behaviour.

Last, to increase specificity for BRD4 and reduce the risk of side effects, Korb et al. tested a low-dose combination of JQ1 and CX-4945, an inhibitor of the kinase that activates BRD4 (casein kinase 2). When used alone at low concentrations, neither drug had discernable effects on experimental mice, but the combination approach showed signs of correcting behavioural deficits in $\mathrm{Fmrl}^{-1-}$ mice.

Together, these findings highlight epigenetic dysregulation and aberrant gene expression as an important disease mechanism downstream of FMRP in FXS, and suggest promising alternative therapeutic targets to synaptic proteins.

Katie Kingwell

ORIGINAL ARTICLE Korb, E. et al. Excess translation of epigenetic regulators contributes to fragile $\mathrm{X}$ syndrome and is alleviated by $\mathrm{Brd} 4$ inhibition. Cell 170, 1209-1223 (2017) 